\title{
Short-Range Nucleon-Nucleon Correlations
}

\author{
Douglas W. Higinbotham \\ Thomas Jefferson National Accelerator Facility, Newport News, VA 23601, USA
}

\begin{abstract}
Valence-shell nucleon knock-out experiments, such as ${ }^{12} \mathrm{C}(\mathrm{e}, \mathrm{e} \cdot \mathrm{p}){ }^{11} \mathrm{~B}$, measure less strength then is predicted by independent particle shell model calculations. The theoretical solution to this problem is to include the correlations between the nucleons in the nucleus in the calculations. Motivated by these results, many electron scattering experiments have tried to isolate the signal from these correlations in order to gain new insight into the short-range part of the nucleon-nucleon potential. Unfortunately, many competing mechanisms can cause the same observable final-state as an initial-state correlation, making truly isolating the signal extremely challenging. This paper reviews the recent experimental evidence for short-range correlations, as well as explores the possibility that such correlations are responsible for the EMC effect in the $0.3<x_{B}<0.7$ deep inelastic scattering ratios.
\end{abstract}

Keywords: Correlations, Nucleon-Nucleon Potential PACS: $13.60 . \mathrm{Hb}, 21.30 .-\mathrm{x}, 21.45 . \mathrm{Bc}$

\section{INTRODUCTION}

The independent particle shell model enjoyed many successes, but failed to predict the strength of valance-shell nucleon knock-out experiments [1,2]. The theoretical solution to this problem was simply to include the correlations that must exist between the nucleons in the nucleus. When compared to an early independent particle shell model calculations, these correlations move strength away from the bound-states and into a continuum of energies and momentums. This effect is illustrated in the work of Benhar et al. where it is shown how the ${ }^{12} \mathrm{C}$ momentum distribution from an independent particle model calculation is reduced at low momentum and enhanced at momentums above $\sim 250 \mathrm{MeV} / \mathrm{c}$ by the addition of nucleon-nucleon correlations [3]. An ab-initio calculation of the depletion of the nuclear Fermi sea by correlations can be found in Ref. [4].

If one could cleanly isolate the high momentum part of the momentum distribution, it would allow electron scattering to provide new information about the short-range part of the nucleon-nucleon potential. This is shown diagrammatically in Fig. 1 where a) shows simple single nucleon knock-out and b) shows the electron knocking-out a correlated pair of nucleons.. Unfortunately, it has proven experimentally challenging to cleanly isolate the effects of short-range correlations from other effects such as meson-exchange currents, isobar-configurations, and final-state interactions. Thus, while the $1981 \mathrm{D}(\mathrm{e}, \mathrm{e}$ 'p)n momentum distribution extraction up to $\approx 250 \mathrm{MeV} / \mathrm{c}$ has stood the test of time [5], that same paper's prediction that one could probe the short-range part of the nucleon-nucleon potential by simply extending the range of kinematics has proven extremely challenging.

\section{ELECTRON SCATTERING KINEMATICS}

The story of the search for nucleon-nucleon correlations is best told with the Brojken $x_{B}$ variable:

$$
x_{B}=\frac{Q^{2}}{2 m \omega}
$$

where $m$ is the mass of the proton, $\omega$ is the energy transfer given by the difference between the beam energy and the scattered electron energy, $Q^{2}$ is the four-momentum transfer given by $\mathbf{q}^{2}-\omega^{2}$ where $\mathbf{q}$ is the three-momentum transfer vector given by the difference between the incident and scattered electron momentum vectors. While this kinematic variable is typically used for deep inelastic physics, it turns out to be a convent variable for counting how many nucleons must be involved in a knock-out reaction. For example, while elastic scattering on a proton has a $x_{B}$ $=1$, quasi-elastic scattering of a nucleon from a nucleus can kinematically extended into the $x_{B}>1$ region due to the presence of the second nucleon. For the $\mathrm{A}\left(\mathrm{e}, \mathrm{e}^{\prime}\right)$ reaction, the $x_{B}$ and $\mathrm{Q}^{2}$ variables can be determined by knowing the properties of the incident beam and measuring the energy and momentum of the scattered electron. 
a)

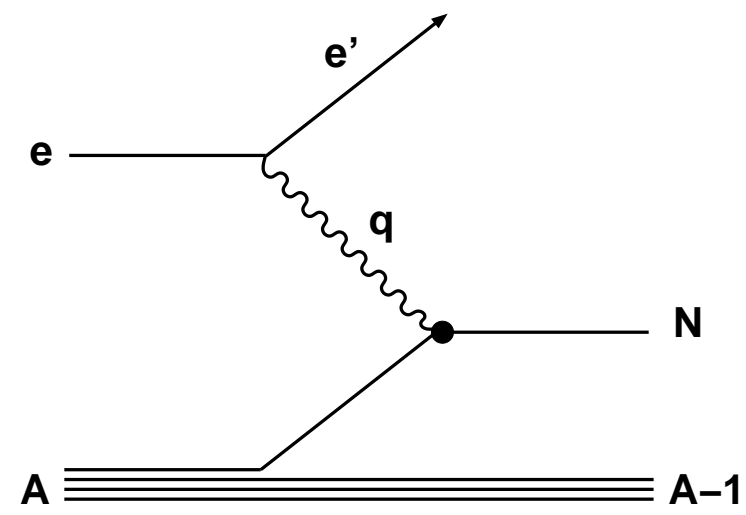

b)

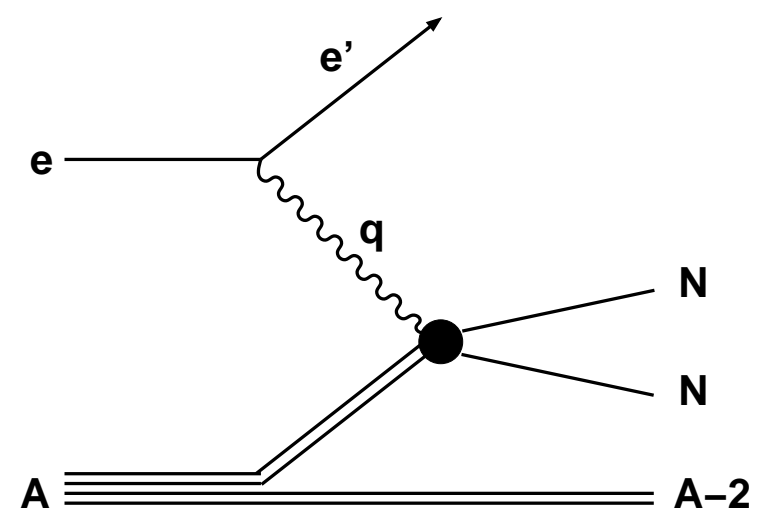

FIGURE 1. The simple goal of short-range nucleon-nucleon correlation studies is to cleanly isolate diagram b) from a). Unfortunately, there are many other diagrams, including those with final-state interactions, that can produce the same final state as the diagram scientists would like to isolate. If one could find kinematics that were dominated by diagram b) it would finally allow electron scattering to provide new insights into the short-range part of the nucleon-nucleon potential.

For A(e,e'p) reactions, one can determine not only the energy and moment transferred, but also the energy and momentum of the knocked-out nucleon. The difference between the transferred and detected energy and momentum is referred to as the missing energy, $E_{\text {miss }}$ and missing momentum, $p_{\text {miss }}$, respectively. From the theoretical works on how short-range nucleon-nucleon correlations effects the momentum distribution of nucleons in the nucleus [6], it is clear one must probe beyond the simple particle in an average potential motion of the nucleon in the nucleus of approximately $250 \mathrm{MeV} / \mathrm{c}$ in order to observe the effects of correlations.

With the construction of the Jefferson Lab Continuous Electron Beam Facility (CEBAF) [7], it was possible to do high-luminosity knock-out reactions in ideal quasi-elastic kinematics into the $p_{\text {miss }}>250 \mathrm{MeV} / \mathrm{c} \mathrm{region}$. In the early Jefferson Lab knock-out reaction proposals, such as E89-044 ${ }^{3} \mathrm{He}\left(\mathrm{e}, \mathrm{e}\right.$ 'p)pn and ${ }^{3} \mathrm{He}(\mathrm{e}, \mathrm{e}$ 'p)d, these kinematics were argued as the key to cleanly observe the effects of short-range correlations. And while final results of the experiments were clearly effected by the presence of correlations, the magnitude of the cross sections in the high missing momentum region was dominated by final-state interaction effects $[8,9]$. Equally striking was the $D\left(e, e^{\prime} p\right) n$ data from CLAS taken at $\mathrm{Q}^{2}>5[\mathrm{GeV} / \mathrm{c}]^{2}$ in $x_{B}<1$ kinematics [10]. Here it was shown that meson-exchange currents, final-state interaction, and delta-isobar configurations mask cleanly probing nucleon-nucleons even at extremely high $\mathrm{Q}^{2}$ in $x_{B}<1$ kinematics.

\section{NUCLEAR SCALING}

With both the $x_{B}<1$ and $x_{B}=1$ kinematics practically ruled out for ever being able to cleanly probe short-range correlations; there is only one region left to explore: $x_{B}>1$. This is a special region, since it is kinematically forbidden for a free nucleon, and thus seems to be a natural place to observe effects of multi-nucleon interactions. These kinematics were probed with limited statistics at SLAC [11] and the plateaus in the per nucleon ratios, r(A/d), were claimed at to be evidence for short-range correlations [12].

In 2003, CLAS published high statics data in the same kinematic region. The results clearly showed that the plateaus could only be seen for $\mathrm{Q}^{2}>1[\mathrm{GeV} / \mathrm{c}]^{2}$ and $x_{B}>1$ kinematics [13] as predicted by Frankfurt and Strikman [14]. But plateaus alone are not evidence for correlations, just evidence that the functional form of the cross section is the same for the two nuclei; so data was taken the $x_{B}>2$ region. By logic, if $1<x_{B}<2$ is a region of two-nucleon correlations, then the $x_{B}>2$ region should be dominated by three-nucleon correlations. The CLAS $\mathrm{Q}^{2}>1$ and $x_{B}>2$ experiment reported observing a second scaling plateau as shown in Fig. 2 [15]. Preliminary results of Hall C high precision data have shown roughly the same magnitude for these plateaus as CLAS and shown that there is no $\mathrm{Q}^{2}$ dependence in the $2<Q^{2}<4[\mathrm{GeV} / \mathrm{c}]^{2}$ range $[16,17]$. 


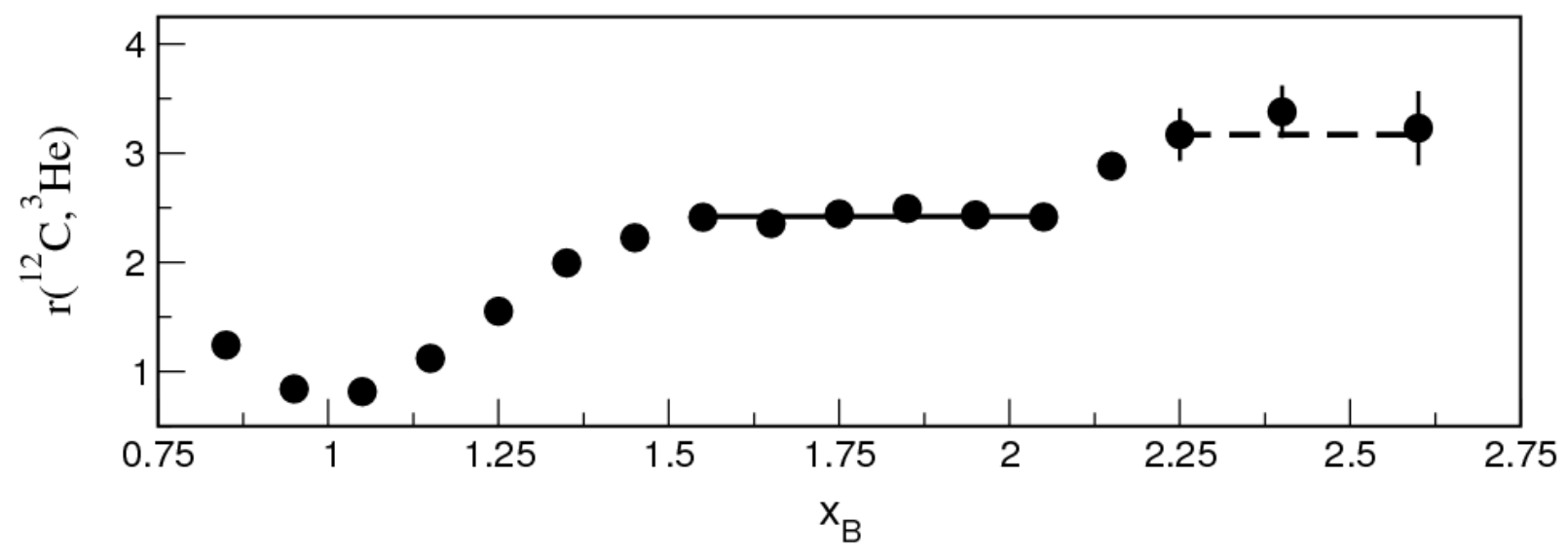

FIGURE 2. Shown is the per nucleon ratio of ${ }^{12} \mathrm{C}\left(\mathrm{e}, \mathrm{e}^{\prime}\right)$ to ${ }^{3} \mathrm{He}\left(\mathrm{e}, \mathrm{e}^{\prime}\right)$ as a function of $x_{B}$ from CLAS [13, 15]. The data show two scaling plateaus. The first, in the $x_{B}>1$ region, is claimed to be due to two-nucleon correlations; while the second, in the $x_{B}>2$ region, is claimed to be due to three-nucleon correlations.

\section{EXCLUSIVE REACTIONS}

While the inclusive results alone are rather convincing, one would like to observe that the final-state is truly dominated by a two-nucleon knock-out process. This can be done by measuring two high-momentum nucleons in the final-state and showing that the rest of the nuclear system is left nearly at rest. Two independent experiments have been carried out that have done this, one at Brookhaven with a proton beam and another at Jefferson Lab with an electron beam.

The Brookhaven experiment made use of a proton beam on a carbon target and detected two forward going protons. Summing the momentum of the two protons and subtracting the incident proton's momentum, allowed them to determined the missing momentum. They then looked for recoiling neutrons with a large acceptance detector. For missing momentum below $250 \mathrm{MeV} / \mathrm{c}$, an isotropic distribution of neutrons were observed; but above $250 \mathrm{MeV} / \mathrm{c}$ the signal was completely dominated by neutrons traveling in the direction of the missing momentum vector $[18,19]$. Analysis of this result showed the reaction was dominated by initial-state neutron-proton pairs [20].

The Jefferson Lab experiment made use of the two Hall A high resolution spectrometers [21] to detect the electron and proton from the ${ }^{12} \mathrm{C}(\mathrm{e}, \mathrm{e} ' \mathrm{p})$ reaction with $\mathrm{Q}^{2}=2[\mathrm{GeV} / \mathrm{c}]^{2}$ and $x_{B}>1$. In the direction of the missing momentum vector, a proton detector known as BigBite and the Hall A Neutron Detector, HAND, were placed in such a way that both recoiling protons and neutron could be detected in nearly the same solid angle acceptance. While the experiment saw the signal of correlated proton-proton pairs [22]; what they discovered was that an overwhelming faction of the events were coming from initial-state neutron-proton pairs [23]. The dominance of neutron-proton paris over protonproton pair was explained by several groups $[24,25,26,27]$ as being due to short-range tensor correlations. In 2011, the experiment will be repeated, but will go to even larger missing momentum. The new experiment expects to finally get into exclusive kinematics where the short-range part of the nucleon-nucleon potential is dominating the reaction.

\section{STRENGTH OF CORRELATIONS IN NUCLEI}

With the exclusive data showing that the inclusive scaling is dominated by initial-state quasi-deuteron neutron-proton correlations, one can confidently use the inclusive scaling results to calculate the strength of the high-momentum part of the momentum distribution for various nuclei. This is done by using a realistic nucleon-nucleon potential [28] to calculate the deuteron's nucleon momentum distribution, $n_{D}(p)$, where the function was normalized such that

$$
1=4 \pi \int_{0}^{\infty} p^{2} \times n_{D}(p) \times d p
$$


TABLE 1. The percent initial-state momentums above different minimum initial momentum values.. The Argonne v-18 nucleon-nucleon potential was used to generate the momentum distribution for the deuteron and the function properly normalized. For the other nuclei, the calculation was only done with momentums greater then $250 \mathrm{MeV} / \mathrm{c}$ where the relation $n(p)_{A}=$ $n(p)_{d} \times a_{2 N}$ can be used and where $a_{2 N}$ is taken from experimental $x_{B}>1$ inclusive nuclear scaling ratios of ${ }^{3} \mathrm{He},{ }^{4} \mathrm{He},{ }^{12} \mathrm{C}$, and ${ }^{56} \mathrm{Fe}$ to deuterium of $2,4,4.8$ and 5.7 , respectively [12, 13].

\begin{tabular}{cccccc}
\hline $\begin{array}{c}\text { Integration Lower Limit } \\
{[\mathbf{M e V} / \mathbf{c}]}\end{array}$ & $\begin{array}{c}\text { Deuteron } \\
{[\boldsymbol{\%}]}\end{array}$ & $\begin{array}{c}\text { Helium-3 } \\
{[\boldsymbol{\%}]}\end{array}$ & $\begin{array}{c}\text { Helium-4 } \\
{[\boldsymbol{\%}]}\end{array}$ & $\begin{array}{c}\text { Carbon-12 } \\
{[\boldsymbol{\%}]}\end{array}$ & $\begin{array}{c}\text { Iron-56 } \\
{[\boldsymbol{\%}]}\end{array}$ \\
\hline 0 & $100^{*}$ & $-\dagger$ & - & - & \\
250 & 5.0 & 10 & 20 & 24 & 28 \\
275 & 4.0 & 8.0 & 16 & 19 & 22 \\
300 & 3.2 & 6.4 & 13 & 15 & 18 \\
370 & 2.5 & 5.0 & 10 & 12 & 15 \\
400 & 2.2 & 4.4 & 8.8 & 10 & 13 \\
440 & 1.8 & 3.6 & 7.2 & 8.6 & 10 \\
500 & 1.3 & 2.6 & 5.2 & 6.2 & 7.4 \\
\hline
\end{tabular}

* Check of proper normalization of the deuteron momentum distribution.

$\dagger$ Many body systems are only calculated above $250 \mathrm{MeV} / \mathrm{c}$ where Eq. 3 should be valid.

. Since the plateaus in the inclusive scaling indicate that the functional forms of the distribution must all have the same shape in this region, the relation for the high momentum part of the distribution can be written

$$
n_{A}(p)=n_{D}(p) \times a_{2 N}
$$

where in this case $a_{2 N}$ is simply the magnitude of the scaling plateau taken from data. To calculate the fraction of the initial-state above a certain momentum for any nuclei where $a_{2 N}$ has been measured, one simply calculates

$$
4 \pi \int_{p_{\min }}^{\infty} p^{2} \times n_{A}(p) \times d p
$$

The integrations of $n_{D}(p)$ and $n_{A}(p)$ starting from various minimum momentums, $p_{\min }$, are shown in Table 1.

\section{THE NUCLEAR EMC EFFECT}

From the results in Table 1, it is clear the magnitude of the high-momentum tail is non-negligible fraction of the initialstate. If this is truly an initial-state effect, then it must appear globally in nuclear physics data. One place to look to see if these numbers provide a solution is to the long standing puzzle of the EMC effect.

Data from the EMC collaboration showed that if one takes ratios of deep-inelastic scattering data from various nuclei one finds structure in the ratios [29]. This is very different from a simple minded picture where one would expect the partons in nucleons to all act the same and the per nucleon ratio to simply be one. Many possible explanations have been put forward to explain this phenomenon [30], yet to this day there is no generally accepted solution.

It is interesting, is to plot EMC type data with a different nuclei in both the numerator and denominator as shown in Fig. 3. There even though the ratio of the number of nuclei is bigger for ${ }^{12} \mathrm{C} /{ }^{4} \mathrm{He}$ and ${ }^{4} \mathrm{He} / \mathrm{D}, 3: 1$ vs $2: 1$, the EMC is dramatically bigger for the ${ }^{4} \mathrm{He} / \mathrm{D}$ ratio. This makes one immediately ask what is nearly the same between ${ }^{12} \mathrm{C}$ and ${ }^{4} \mathrm{He}$, but very different for ${ }^{4} \mathrm{He}$ and $\mathrm{D}$. One obvious answer is the average binding energy; but another answer, related to the first, is the strength of short-range correlations. In fact, as pointed out in Refs. [31, 32], the magnitude of the EMC effect seems to follow the magnitudes of the nuclear scaling plateaus. This phenomenological correlation between the EMC effect and the scaling plateaus is shown in Fig. 4. Assuming this correlation is showing the reason for the EMC effect, it is even possible to use the magnitudes of the scaling plateaus to correct the deuteron back to the case of a free proton and neutron allowing for a phenomenological extraction of the F2n structure function [32]. 

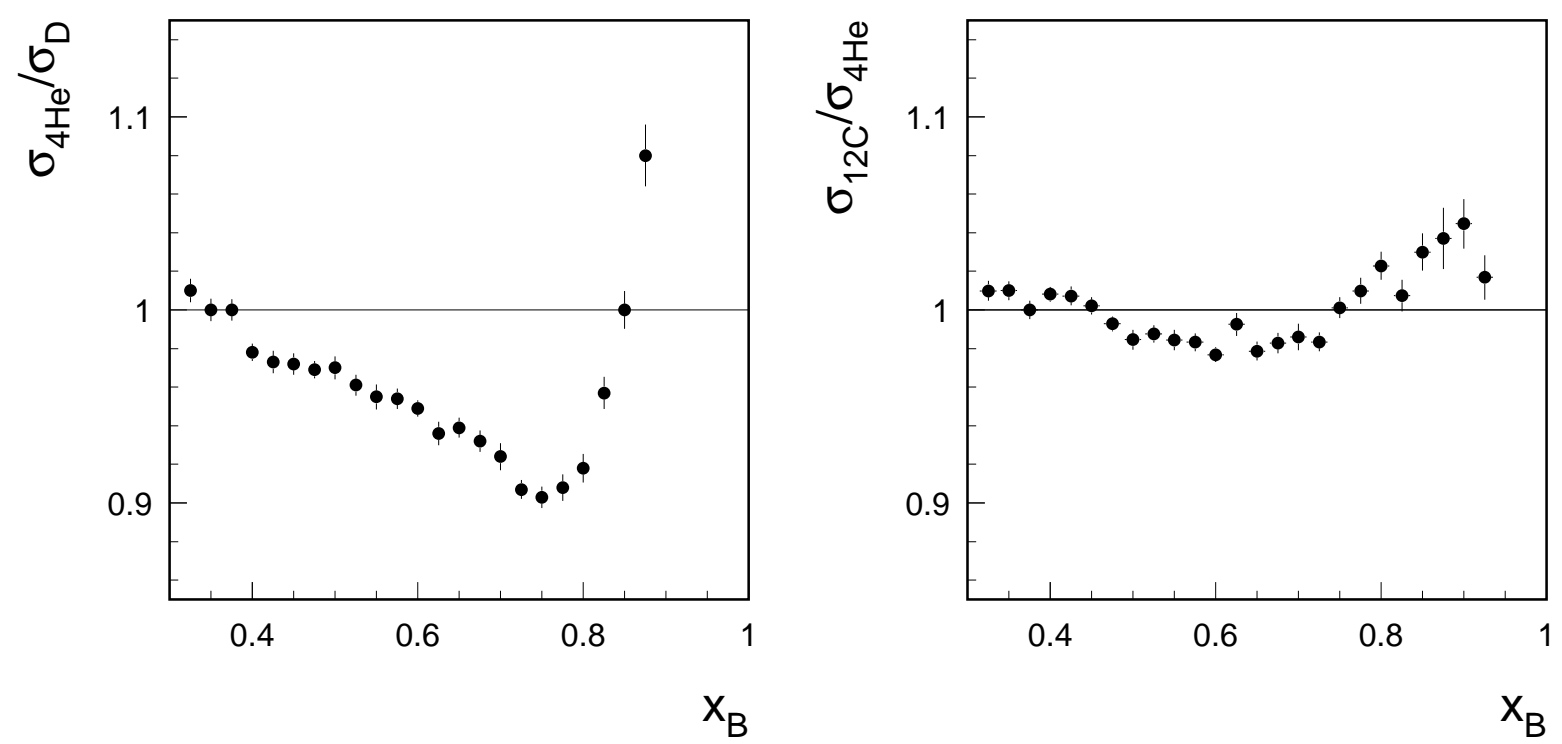

FIGURE 3. Shown on the left is the Hall $\mathrm{C}$ deep inelastic per nucleon cross section ratio of ${ }^{4} \mathrm{He}$ to $\mathrm{D}$ with its characteristic dip around $x_{B}$ of 0.75 [33]. On the other hand, the ratio of ${ }^{12} \mathrm{C}$ to ${ }^{4} \mathrm{He}$ the effect is relatively small, which is consistent with the claim EMC is a local density effect.

\section{SUMMARY}

After years of trying to cleanly probe short-range correlations, recent $\mathrm{Q}^{2}>1[\mathrm{GeV} / \mathrm{c}]^{2}$ and $x_{B}>1$ experiments seem to have finally isolated the signal. The beauty is not from any one result, but from the fact that all of the new data seems to be pointing at the same underlying mechanism. In fact, with the the magnitude of short-range correlations in hand, it seems to provide a phenomenological explanation for the $0.3<x_{B}<0.7$ EMC effect slope and provide a phenomenological way of extracting the neutron's deep inelastic structure function.

\section{ACKNOWLEDGMENTS}

Special thanks to Eli Piatetzky for getting me involved in short-range nucleon-nucleon studies and also for suggesting to the organizers I present this talk. I also thank Javier Gomez, Larry Weinstein, Mark Strikman, Jerry Miller, Franz Gross, Wally van Orden, and Misak Sargsian for the many interesting discussions about what these new experimental results are trying to tell us. This work was supported by the U.S. Department of Energy and Jefferson Science Associates which operates the Thomas Jefferson National Accelerator Facility under DOE contract DE-AC05$06 \mathrm{OR} 23177$.

\section{REFERENCES}

1. L. Lapikas, Nucl. Phys. A553, 297-308 (1993).

2. J. J. Kelly, Adv. Nucl. Phys. 23, 75-294 (1996).

3. O. Benhar, D. day, and I. Sick, Rev. Mod. Phys. 80, 189-224 (2008), nucl-ex/0603029.

4. A. Rios, A. Polls, and W. H. Dickhoff, Phys. Rev. C79, 064308 (2009), arXiv : 0904.2183.

5. M. Bernheim, et al., Nucl. Phys. A365, 349-370 (1981).

6. C. Ciofi degli Atti, and S. Simula, Phys. Rev. C53, 1689 (1996), nucl-th/ 9507024.

7. C. Leemann, D. Douglas, and G. Krafft, Ann.Rev.Nucl.Part.Sci. 51, 413-450 (2001).

8. F. Benmokhtar, et al., Phys. Rev. Lett. 94, 082305 (2005), nucl-ex/ 0408015.

9. M. M. Rvachev, et al., Phys. Rev. Lett. 94, 192302 (2005), nucl-ex/ 0409005.

10. K. S. Egiyan, et al., Phys. Rev. Lett. 98, 262502 (2007), nucl-ex/ 0701013.

11. D. B. Day, J. S. McCarthy, T. W. Donnelly, and I. Sick, Ann. Rev. Nucl. Part. Sci. 40, 357-410 (1990).

12. L. L. Frankfurt, M. I. Strikman, D. B. Day, and M. Sargsian, Phys. Rev. C48, 2451-2461 (1993). 


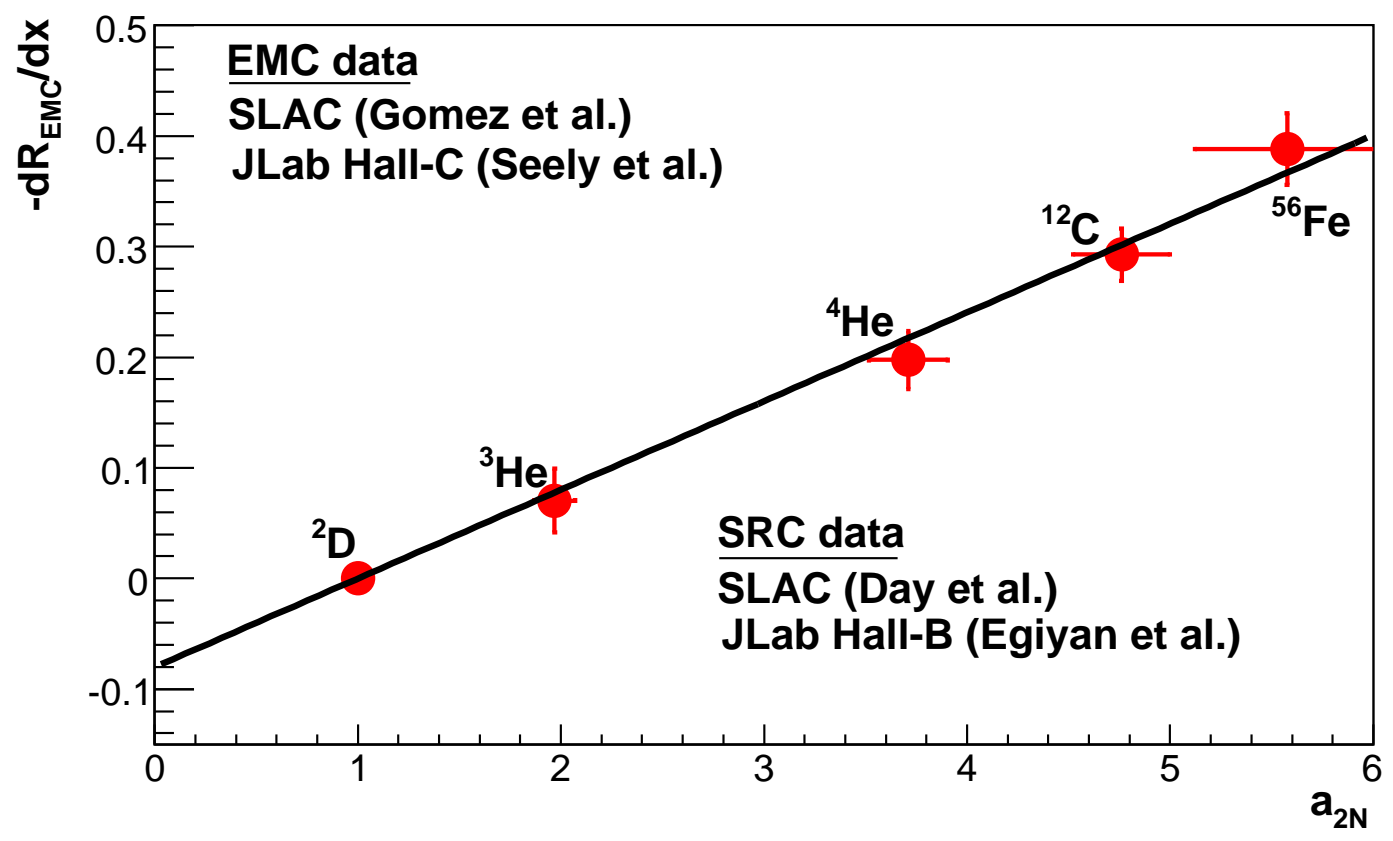

FIGURE 4. Shown is the $0.3<x_{B}<0.7$ EMC slopes, $\mathrm{dR} / \mathrm{dx}$, for various nuclei from Seely and Gomez [33, 34] plotted vs the magnitude of the $x_{B}>1$ nuclear scaling ratio, $a_{2 N}$, for various nuclei from Day and Egiyan [11, 13]. The linear correction between these two seemly disconnected regions is already striking and soon to be published data will further enhance the results [16].

13. K. S. Egiyan, et al., Phys. Rev. C68, 014313 (2003), nucl-ex/ 0301008.

14. L. L. Frankfurt, and M. I. Strikman, Phys. Rept. 76, 215-347 (1981).

15. K. S. Egiyan, et al., Phys. Rev. Lett. 96, 082501 (2006), nucl-ex/0508026.

16. N. Fomin, AIP Conf. Proc. 947, 174-177 (2007), arXiv: 0808.2625.

17. N. Fomin (2008), Ph.D.Thesis, arXiv: 0812.2144.

18. A. Tang, et al., Phys. Rev. Lett. 90, 042301 (2003), nucl-ex/0206003.

19. J. Watson, AIP Conf.Proc. 1056, 295-300 (2008).

20. E. Piasetzky, M. Sargsian, L. Frankfurt, M. Strikman, and J. W. Watson, Phys. Rev. Lett. 97, 162504 (2006), nucl-th/0604012.

21. J. Alcorn, et al., Nucl. Instrum. Meth. A522, 294-346 (2004).

22. R. Shneor, et al., Phys. Rev. Lett. 99, 072501 (2007), nucl-ex/ 0703023.

23. R. Subedi, et al., Science 320, 1476-1478 (2008), arXiv: 0908 . 1514.

24. R. Schiavilla, R. B. Wiringa, S. C. Pieper, and J. Carlson, Phys. Rev. Lett. 98, 132501 (2007), nucl-th/0611037.

25. M. M. Sargsian, T. V. Abrahamyan, M. I. Strikman, and L. L. Frankfurt, Phys. Rev. C71, 044615 (2005), nucl-th/ 0501018.

26. R. B. Wiringa, R. Schiavilla, S. C. Pieper, and J. Carlson, Phys. Rev. C78, 021001 (2008), arXiv: 0806.1718.

27. M. Alvioli, C. Ciofi degli Atti, and H. Morita, Phys. Rev. Lett. 100, 162503 (2008).

28. R. B. Wiringa, V. G. J. Stoks, and R. Schiavilla, Phys. Rev. C51, 38-51 (1995), nucl-th/ 9408016.

29. J. Aubert, et al., Phys.Lett. B123, 275 (1983).

30. D. F. Geesaman, K. Saito, and A. W. Thomas, Ann. Rev. Nucl. Part. Sci. 45, 337-390 (1995).

31. D. W. Higinbotham, J. Gomez, and E. Piasetzky, submitted to Phys. Lett. B (2010), arXiv: 1003.4497.

32. L. B. Weinstein, et al., submitted to Phys. Rev. Lett. (2010), arXiv:1009.5666.

33. J. Seely, et al., Phys. Rev. Lett. 103, 202301 (2009), arXiv: 0904.4448.

34. J. Gomez, R. Arnold, P. E. Bosted, C. Chang, A. Katramatou, et al., Phys.Rev. D49, 4348-4372 (1994). 